\title{
Combined use of xenogeneic bone substitute material covered with a native bilayer collagen membrane for alveolar ridge preservation: A randomized controlled clinical trial
}

\author{
Jung, Ronald E ; Sapata, Vitor M ; Hämmerle, Christoph H F ; Wu, Hui ; Hu, Xiu-Lian ; Lin, Ye
}

\begin{abstract}
AIM The aim of this split-mouth randomized controlled study was to evaluate radiographic dimensional changes after tooth extraction in posterior sites treated with a ridge preservation technique or left for spontaneous healing. MATERIALS AND METHODS In a total of 18 patients, tooth extraction in posterior sites of the upper and lower jaw was performed in a split-mouth design. The post-extraction sockets were randomly assigned to the following two treatment modalities: deproteinized bovine bone mineral (DBBM) with $10 \%$ collagen (DBBM-C) covered with a native bilayer collagen membrane (NBCM) (test group) and spontaneous healing (control group). Cone beam computed tomography (CBCT) scans were performed after extractions, 3 and 6 months later. The following parameters were measured: the height of the buccal bone plate $(\mathrm{BH})$, height of the palatal bone plate $(\mathrm{PH})$, horizontal width of the extraction socket at $1 \mathrm{~mm}, 3 \mathrm{~mm}$, and $5 \mathrm{~mm}(\mathrm{HW}-1, \mathrm{HW}-3, \mathrm{HW}-5)$, and the horizontal width (thickness) of the buccal bone plate at $1 \mathrm{~mm}, 3 \mathrm{~mm}$, and $5 \mathrm{~mm}$ (BHP-1, BHP-3, BHP-5). Statistical analysis was performed applying a nonparametric Wilcoxon signed-rank test. RESULTS The CBCT analysis showed a bone loss compared to baseline in test and control group. The measurements which have reached statistically significant differences at 6 months were $\mathrm{BH}$ (test: $-2.31 \%$ vs control: $-13.11 \%$ ), $\mathrm{PH}$ (test: $-2.07 \%$ vs control: 15.32\%), HW-1 (test: $-17.14 \%$ vs control: $-32.47 \%$ ), and HW-3 (test: $-11.65 \%$ vs control: $-28.47 \%$ ). CONCLUSIONS The posterior ridge preservation technique using DBBM-C covered with a NBCM is a valid approach reducing the amount of the radiographic loss in alveolar ridge dimensions.
\end{abstract}

DOI: https://doi.org/10.1111/clr.13149

Posted at the Zurich Open Repository and Archive, University of Zurich

ZORA URL: https://doi.org/10.5167/uzh-150983

Journal Article

Accepted Version

Originally published at:

Jung, Ronald E; Sapata, Vitor M; Hämmerle, Christoph H F; Wu, Hui; Hu, Xiu-Lian; Lin, Ye (2018). Combined use of xenogeneic bone substitute material covered with a native bilayer collagen membrane for alveolar ridge preservation: A randomized controlled clinical trial. Clinical Oral Implants Research, 29(5):522-529.

DOI: https://doi.org/10.1111/clr.13149 
Combined use of xenogeneic bone substitute material covered with a native bilayer collagen membrane for alveolar ridge preservation: A randomized controlled clinical trial

Jung $\mathrm{RE}^{1}$, Sapata $\mathrm{VM}^{1}$, Hämmerle, $\mathrm{CHF}^{1}$, Hui Wu${ }^{2}$, Xiu-lian $\mathrm{Hu}^{2}$, Ye Lin²

${ }^{1}$ Clinic of Fixed and Removable Prosthodontics and Dental Material Science, University of Zurich, Zurich, Switzerland

${ }^{2}$ Implant Center, Peking University School and Hospital of Stomatology, Peking, China

Key words: bone regeneration; bone substitute; cone beam computed tomography; ridge preservation.

Running title: Posterior ridge preservation

Address for correspondence:

Ronald E. Jung, Prof. Dr. med. dent., PhD

Clinic of Fixed and Removable Prosthodontics and Dental Material Science

University of Zurich

Plattenstrasse 11

CH-8032 Zurich, Switzerland

Phone: +41446343260

Fax: +41446344305

e-mail: ronald.jung@zzm.uzh.ch 


\section{Abstract}

Aim: the aim of the present split-mouth randomized controlled study was to evaluate radiographic dimensional changes after tooth extraction in posterior sites treated with a ridge preservation technique or left for spontaneous healing. Materials and Methods: In a total of 18 patients tooth extraction in posterior sites of the upper and lower jaw was performed in a split-mouth design. The post-extraction sockets were randomly assigned to the following two treatment modalities: deproteinized bovine bone mineral (DBBM) with $10 \%$ collagen (DBBM-C) covered with a native bilayer collagen membrane (NBCM) (test group) and spontaneous healing (control group). Cone Beam Computed Tomography (CBCT) scans were performed after extractions, 3 and 6 months later. The following parameters were measured: the height of the buccal bone plate $(\mathrm{BH})$, height of the palatal bone plate $(\mathrm{PH})$, horizontal width of the extraction socket at $1 \mathrm{~mm}, 3 \mathrm{~mm}$, and $5 \mathrm{~mm}(\mathrm{HW}-1, \mathrm{HW}-3, \mathrm{HW}-5)$ and the horizontal width (thickness) of the buccal bone plate at $1 \mathrm{~mm}, 3 \mathrm{~mm}$, and $5 \mathrm{~mm}$ (BHP-1, BHP-3, BHP-5). Statistical analysis was performed applying a nonparametric Wilcoxon signed rank test. Results: The $\mathrm{CBCT}$ analysis showed a bone loss compared to baseline in test and control group. The measurements which have reached statistically significant differences at 6 months were $\mathrm{BH}$ (test: $-2.31 \%$ vs. control: $-13.11 \%), \mathrm{PH}$ (test: $-2.07 \%$ vs. control: $-15.32 \%), \mathrm{HW}-1$ (test: $-17.14 \%$ vs. control: $-32.47 \%$ ), and HW-3 (test: $-11.65 \%$ vs. control: $-28.47 \%)$. Conclusions: The posterior ridge preservation technique using DBBM-C covered with a NBCM is a valid approach reducing the amount of the radiographic loss in alveolar ridge dimensions. 


\section{Introduction}

After tooth extraction, spontaneous healing process causes bone remodeling and consequently shape and volume loss of the initial socket contour (Cardaropoli et al., 2003; Araujo \& Lindhe, 2005; Hämmerle et al., 2012; Araújo et al., 2015b) The remodeling process starts immediately after tooth extraction, and after 2 years an average of $40-60 \%$ bone loss is detected in vertical and horizontal dimensions (Johnson, 1969; Tan et al., 2012). This amount of bone resorption in the alveolar process may interfere with the placement of dental implants.

Alveolar ridge preservation technique (ARP) has been proposed to reduce the bone resorption after tooth extraction (Darby et al., 2009; MacBeth et al., 2017). Several studies evaluated the ARP technique using different bone graft materials such as autogenous bone (Becker et al., 1994), demineralized freeze-dried bone allograft (Froum et al., 2002), mineralized freeze-dried bone allograft (Feuille et al., 2003), deproteinized bovine bone (Artzi et al., 2000), alloplastic polymers (Serino et al., 2003) and bioactive glasses (Froum et al., 2002). From all these bone graft materials deproteinized bovine bone (DBBM) might be the most often used material for ARP in the past years (Araújo et al., 2015a). In recent randomized clinical trials (RCT), it has been demonstrated that DBBM with $10 \%$ collagen for ridge preservation in anterior sites (Jung et al., 2013; Meloni et al., 2015; Araújo et al., 2015b) reduced the radiographic bone resorption.

However, the majority of these clinical studies focus on anterior single rooted teeth, ignoring the posterior region of the jaws, where the location of the maxillary 
sinus or mandibular nerve limits the installation of dental implants.

Hence, the aim of the present split-mouth randomized controlled study was to evaluate, through Cone Beam Computed Tomography (CBCT) analysis, the dimensional changes after tooth extraction in posterior sites either treated with a ridge preservation technique or left for spontaneous healing. 


\section{Materials \& Methods}

\section{$\underline{\text { Study design }}$}

This study is a prospective, randomized, controlled, clinical trial in a split-mouth design according to the Helsinki protocol. All procedures and materials were approved by the ethical committee of Peking University School and Hospital of Stomatology, China (Ref. № PKUSSIRB-2012003). Twenty-four patients that visited the implant center of Peking University School and Hospital of Stomatology were included.

Inclusion criteria

i) Age: > 18 years;

ii) Need of symmetric molar or premolar extraction within the same jaw;

iii) Presence of one adjacent tooth at the extraction site;

iv) Adequate oral hygiene (bleeding on probing $<20 \%$; plaque index $<20 \%$ );

v) Systemically healthy with no contraindication for oral surgical procedures;

vi) Signed informed consent form.

\section{Exclusion criteria}

i) Pregnant or lactating women;

ii) Existence of bone metabolic disease;

iii) Currently taking drugs that influence bone metabolism;

iv) Use of bisphosphonates in the last 4 years;

v) Smokers (>10 cigarettes/day). 


\section{$\underline{\text { Randomization }}$}

A computer-generated list was prepared (http://www.randomizer.org/) and concealed randomization envelopes were stored by an independent person unaware of the study protocol. The extraction sockets within the same jaw of each patient were randomly assigned to the test group and the control group.

\section{Surgical procedure}

An experienced oral surgeon did all the extractions and alveolar ridge preservations. Extractions were performed under local anesthesia. Periotomes and elevators were used with great care to preserve the buccal bone plate and the surrounding soft and hard tissues. When it was necessary, the teeth were sectioned by a handpiece with diamond burs. No flap was elevated. After tooth extraction, the sockets were carefully debrided to remove all soft tissue remnants. Thereafter, the extraction sockets were randomly assigned to the following two treatment modalities:

i) Test group: the sockets were completely filled with deproteinized bovine bone mineral with $10 \%$ collagen (DBBM-C; Geistlich Bio-Oss ${ }^{\circledR}$ Collagen, Geistlich Pharma AG, Wolhusen, Switzerland), then covered with a native bilayer collagen membrane (NBCM; Geistlich Bio-Gide ${ }^{\circledR}$, Geistlich Pharma AG, Wolhusen, Switzerland). The membrane was shaped in a way to overlap the margins of the extraction sockets by $2-3 \mathrm{~mm}$ and placed slightly underneath 
the marginal mucosa. Subsequently, a cross suture was performed to keep the membrane in place.

ii) Control group: the sockets healed spontaneously without any graft materials and with no sutures.

All the patients were instructed to rinse with $0.12 \%$ chlorhexidine for 1 minute, twice a day, for ten days. The sutures of the test site were removed 7-10 days after extraction.

\section{$\underline{\text { Radiographic analysis }}$}

Cone Beam Computed Tomography (CBCT) was performed at baseline (immediately after teeth extraction), 3 months and 6 months after tooth extraction. The Field of View (FOV) of a cylinder measuring $8 \times 8 \mathrm{~cm}$ was selected and an image was acquired with $0.16 \mathrm{~mm}$ of resolution (Planmeca Promax, Planmeca Oy, Finland). The scan datasets (DICOM, Digital Imaging and Communications in Medicine) were compared using an open-source software (3D Slicer 4.5, www.slicer.org). The datasets were matched utilizing areas where no changes had taken place during the follow-up periods (e.g. the cranial base - maxilla - and the mandible - external and internal oblique ridge). Subsequently, images with an embedded ruler were taken in the center of the extraction socket of the mesial and distal roots at each time point. An image-editing software program (Adobe Photoshop CS6, Adobe Systems Incorporated, USA) was used to layer and delimit the following points at baseline according to figure 1 . The most apical point of mesial and distal extraction socket, a 
vertical reference line - parallel to the long axis of the tooth, or it's adjacent tooth - in the center of the extraction socket (crossing the apical reference point), two horizontal reference lines (perpendicular to the vertical line, crossing the top of the lingual/palatal bone plate and the most apical point of the extraction socket) and three reference lines with dots on the lingual/palatal wall of the root (at $1 \mathrm{~mm}, 3 \mathrm{~mm}$ and $5 \mathrm{~mm}$ below the horizontal reference line) (Fig. 1). Another set of images from each patient were exported. Therefore, the measurements were made using the same reference points and lines defined in the baseline image for the 3 and 6 months follow-up. This method of analysis has been adapted according to a previous clinical study assessing single rooted tooth in the anterior region (Jung et al. 2013). The following images were calibrated with the embedded ruler and the measurements were taken using ImageJ software (ImageJ, Image J64, National Institute of Health, USA) by a single calibrated evaluator (V.M.S.):

i) The Height of the Buccal $(\mathrm{BH})$ and Palatal (PH) bone plate at baseline, 3 and 6 months' follow-up.

ii) The Horizontal Width of the extraction socket measured at $1 \mathrm{~mm}, 3 \mathrm{~mm}$ and $5 \mathrm{~mm}$ below the lingual bone crest at baseline, 3 and 6 months' follow-up (HW-1, HW-3, HW-5);

iii) The Horizontal Width (thickness) of the buccal bone plate at $1 \mathrm{~mm}, 3 \mathrm{~mm}$, and $5 \mathrm{~mm}$ below the lingual bone crest at baseline (BHP-1, BHP-3, BHP-5);

\section{Statistical analysis}

The primary outcome of the present study was the Horizontal Width of the 
extraction socket measured at $1 \mathrm{~mm}(\mathrm{HW}-1)$, with patient being the unit of analysis. The sample size was based on a previous publication (Jung et al., 2013), considering a standard deviation of $0.7 \mathrm{~mm}$ and a mean outcome of $1.1 \mathrm{~mm}$ for control and $0.6 \mathrm{~mm}$ for test group. The significance level was set at $5 \%$ and power of $60 \%$. Twenty-four patients were included, considering a 20\% drop-out rate. Intra-rater reliability was assessed by re-measuring more than 24 individual measurements from 10 patients. The corresponding intra-class correlation (ICC) and confidence intervals were calculated. Intra-rater reliability was excellent as confidence intervals for ICC were all above 0.95 for all parameters. The raw data have been checked for differences between the mesial and distal root. As there was no difference the dataset was first averaged over different roots of the same tooth, providing an average test and control value per patient. Descriptive statistics (mean value and standard deviation) were obtained and a nonparametric Wilcoxon signed rank test for paired data was employed to check for significant differences between the test and control groups. Tests and plots were done with the software R (R Core Team, 2015) and the significance level was set to alpha $=0.05$. 


\section{Results}

A total of 24 patients were treated in the present study. During the healing period no graft loss or infection occurred. Four patients were excluded due to artifacts in the CBCT scans and two patients were excluded due to the fact, that it was impossible to set the reference lines. Finally, 18 patients with a split-mouth design were included for analysis (12 patients with upper jaw sites - 12 upper pre-molar, 12 upper molar - and 6 patients with lower jaw sites - 4 lower pre-molar, 8 lower molar) (Fig. 2 \& 3).

The data are presented in figure 4. The CBCT analysis showed, in all measurements, a bone loss compared to baseline. The measurements which reached statistically significant differences between test and control group at 3 months were the $\mathrm{BH}$ (test: $-1.96 \%$ / $-0.56 \mathrm{~mm}$ vs. control: $-11.80 \%$ / $-1,41 \mathrm{~mm}$ ), height of $\mathrm{PH}$ (test: $-1.52 \%$ / - $0.56 m m$ vs. control: $-13.21 \%$ / $-1.16 m m$ ) and HW-3 (test: $-7.81 \%$ / $-0.72 m m$ vs. control: $-21.44 \%$ / $-1.84 \mathrm{~mm})$. At 6 months, statistically significant differences between the test and control group were found in a vertical dimension at $\mathrm{BH}$ (test: $-2.31 \%$ / $-0.25 m m$ vs. control: $-13.11 \% /-1.25 m m$ ) and PH (test: $-2.07 \% /-0.26 m m$ vs. control: $-15.32 \%$ / $-0.89 \mathrm{~mm}$ ) (Fig. 4a), and at a horizontal dimension at HW-1 (test: $-17.14 \%$ / -1.33mm vs. control: $-32.47 \%$ / $-2.78 m m$ ) and HW-3 (test: $-11.65 \%$ / $-0.82 \mathrm{~mm}$ vs. control: $-28.47 \% /-1.95 \mathrm{~mm}$ ) (Fig. $4 \mathrm{~b})$.

The percentage of bone loss in the upper jaw are presented in table 1 . The measurements which have reached statistically significant differences between test and control group at 3 months were $\mathrm{BH}$ (test: $-1.75 \%$ / $-0.78 \mathrm{~mm}$ vs. control: $-7.67 \%$ / 
$-1.26 \mathrm{~mm}$ ), PH (test: $-1.38 \%$ / -0,79mm vs. control: $-10.35 \% /-0.94 \mathrm{~mm}$ ) and HW-3 (test: $-6.72 \%$ / $-0.87 \mathrm{~mm}$ vs. control: $-18.07 \% /-1.61 \mathrm{~mm}$ ) and at 6 months, were $\mathrm{BH}$ (test: $-2.06 \%$ / $-0.32 \mathrm{~mm}$ vs. control: $-7.17 \%$ / $-0.84 \mathrm{~mm}$ ) and HW-3 (test: $-11.73 \%$ / $-0.91 \mathrm{~mm}$ vs. control: $-20.82 \% /-1.33 \mathrm{~mm})$.

The percentage of bone loss in the lower jaw are presented in table 2 . The measurements which have reached statistically significant differences between test and control group at 3 months were BH (test: $-2.95 \%$ / $-0.15 m m$ vs. control: $-20.23 \%$ / -1.66mm) and PH (test: $-2.16 \% /-0.15 m m$ vs. control: $-18.83 \% /-1.53 m m$ ) and at 6 months were $\mathrm{BH}$ (test: $-2.18 \% /-0.12 \mathrm{~mm}$ vs. control: $-23.7 \% /-1.96 \mathrm{~mm}$ ), $\mathrm{PH}$ (test: $-2.46 \%$ / -0.17mm vs. control: $-19.84 \%$ / $-1.60 \mathrm{~mm}$ ), HW-3 (test: $-12.53 \% /-0.67 m m$ vs. control: $-43.74 \%$ / -2.97mm) and HW-5 (test: $-5.07 \%$ / -0.21mm vs. control: $-23.51 \%$ / $-1.24 \mathrm{~mm})$

The relationship between BHP-1 and the bone loss is presented in the following plots (Fig. 5). Independent of the treatment group no major bone loss (e.g. more than 30\%) was observed when the buccal bone plate was wider than $1 \mathrm{~mm}$. 


\section{Discussion}

The present $\mathrm{CBCT}$ analysis in posterior sites of both jaws demonstrated an overall reduction of the radiographic bone resorption after tooth extraction. However, the use of DBBM-C covered with a NBCM apparently reduced the radiographic loss in alveolar ridge dimensions in the horizontal and vertical direction, with statistically significantly less bone resorption at $\mathrm{BH}, \mathrm{PH}$, and $\mathrm{HW}-3$.

There is a large body of literature evaluating the dimensional changes of the alveolar ridge in the anterior area after tooth extraction (Tan et al., 2012; Avila-Ortiz et al., 2014; Mardas et al., 2015; MacBeth et al., 2017). Recent randomized controlled clinical trials (RCTs) using xenografts for post-extraction socket preservation demonstrated a significant reduction of the alveolar bone contour in anterior sites (Jung et al. 2013; Araújo et al. 2015b; Meloni et al. 2015). One of these RCTs involving 40 patients, showed a reduction of $43 \%$ in the spontaneous healing group compared to $18 \%$ of xenograft groups at $1 \mathrm{~mm}$ below the crest (Jung et al., 2013). Another RCT with 48 anterior extraction sockets assessing spontaneous healing vs. alveolar ridge preservation with xenograft demonstrated a considerable reduction in the amount of horizontal and vertical bone resorption in the xenograft group (Cardaropoli et al., 2013). A recent RCT compared extraction sockets that have been preserved with the same xenograft but covered either by a porcine collagen matrix or an epithelial connective tissue graft. It has been concluded that both treatment modalities showed favorable and similar outcomes, however, the use of the porcine collagen matrix allowed simplification of the treatment because no 
palatal donor site was involved (Meloni et al., 2015).

In contrast to the previously mentioned literature on alveolar ridge preservation procedures (ARP) in anterior sites, the present RCT assessed exclusively posterior sites after tooth extraction. Due to the anatomic and morphologic differences, it seems that posterior sites demonstrate a different pattern of bone remodeling after tooth extraction. Considering the buccal bone plate height $(\mathrm{BH})$, there is more resorption in posterior sites (Test: $-2.31 \%$ posterior vs. $-0.1 \%$ anterior; Control: $-13.11 \%$ posterior vs. $-5.5 \%$ anterior) compared to anterior single rooted sites (Jung et al. 2013). The changes taking place in the horizontal dimension of the extraction socket at $1 \mathrm{~mm}(\mathrm{HW}-1)$ below the crest demonstrated similar results in anterior and posterior sites receiving $\operatorname{ARP}(-17.14 \%$ posterior vs. $-17.4 \%$ anterior). However, unassisted sockets healing in the posterior area revealed less resorption compared to the anterior region (-32.47\% posterior vs. $-43.3 \%$ anterior) (Jung et al., 2013). Due to the larger dimensions in width compared to the anterior sites and the non-aesthetic appeal, the greater resorption in posterior sites might not cause a big impact for posterior implant placement. However, it might be speculated that the significantly higher amount of resorption in the vertical dimension could lead to more augmentative procedures during or before implant placement and, therefore, to more complex procedures and to an increased morbidity for sites receiving no ARP. Moreover, a recent RCT (Walker et al., 2017) evaluating posterior ridge preservation also demonstrate a higher radiographic bone resorption at sites without ridge preservation procedure, especially at the buccal aspect. 
Therefore, one of the main advantages of posterior ridge preservation, especially in the upper jaw, could be to avoid secondary bone regeneration procedures (e.g. minor GBR procedures, sinus elevation) to place implants. A systematic review (Mardas et al., 2015) showed a decreased necessity for further augmentation procedures when ridge preservation was performed in anterior sites (Relative risk: 0.15, 95\% Cl: 0.07-0.3). Another recent RCT also demonstrated a reduced necessity of GBR procedure at implant placement in posterior sites when ridge preservation was performed $(10 \%$ of implants needed GBR at implant placement at ridge preservation group against $25 \%$ on control group) (Walker et al., 2017). The test group of the present study showed significantly less bone resorption on the height of buccal and palatal bone wall compared to the control group (BH Test: $-2.31 \%$ vs. BH Control: -13.11\%; PH Test: $-2.07 \%$ vs. PH Control: $-15.32 \%)$. Another important point is the relation between the maxillary sinus and the tip of the roots. Cases with a close relationship between the sinus and the tip of the root seem to suffer more from vertical resorption because of the sinus pneumatization (Sharan \& Madjar, 2008). This has not been documented in the present split-mouth randomized clinical trial. The evaluated Chinese population presented a generous amount of bone between the tip of the root and the floor of the sinus (Fig. 2).

The correlation analysis between the horizontal width (thickness) of the buccal bone plate at $1 \mathrm{~mm}(\mathrm{BHP}-1)$ and the other parameters demonstrated a possible direct influence on the radiographic bone resorption rate. Cases with more than $1 \mathrm{~mm}$ of BHP-1 did not suffer extreme bone loss (e.g. more than 30\%) in both groups. However, 
the ridge preservation technique prevented a major bone remodeling, especially in height, even in cases with less than $1 \mathrm{~mm}$ of BHP-1.

The limitations of the present study were the low number of patients included, reducing the power of the present study to $60 \%$, and consequently rising the chance of an "false negative" (Type II error). The difficulties regarding the CBCT analysis to identify the bone graft material due to the similar density compared to the other tissues in the extraction socket and the recurrent presence of artifacts in the CBCT scans (e.g. metal restoration, dental implants, etc.) were also considered a limitation of the present study. Although several studies have used native bilayer collagen membrane to seal the extraction socket, a possible drawback of the present study could be the use of this type of membrane, with an earlier breakdown compared to a non-cross-linked type I/III collagen-based material. The Chinese population is also a concern to the authors, as the anatomic characteristics are very singular and maybe not found in other populations. Moreover, there is a need for further RCTs comparing the ridge alterations after tooth extraction in posterior sockets. The necessity for secondary bone regeneration and the sinus floor alterations should be evaluated. 


\section{Conclusions}

The posterior ridge preservation technique, using DBBM-C covered with a NBCM, could be a valid approach reducing the amount of radiographic loss in alveolar ridge dimensions in the posterior area. Statistically significant differences were found between test and control group in bone height (buccal and palatal/lingual) and bone width ( 1 and $3 \mathrm{~mm}$ below the bone crest) 6 months after tooth extraction, in favor of the posterior ridge preservation technique. 


\section{References}

Araujo M.G. \& Lindhe J. (2005) Dimensional ridge alterations following tooth extraction. An experimental study in the dog. Journal of Clinical Periodontology 32: 212-218.

Araújo M.G., Silva C.O., Misawa M. \& Sukekava F. (2015a) Alveolar socket healing: what can we learn? Periodontology 2000 68: 122-134.

Araújo M.G., da Silva J.C.C., de Mendonça A.F. \& Lindhe J. (2015b) Ridge alterations following grafting of fresh extraction sockets in man. A randomized clinical trial. Clinical Oral Implants Research 26: 407-412.

Artzi Z., Tal H. \& Dayan D. (2000) Porous bovine bone mineral in healing of human extraction sockets. Part 1: histomorphometric evaluations at 9 months. Journal of Periodontology 71: 1015-1023.

Avila-Ortiz G., Elangovan S., Kramer K.W.O., Blanchette D. \& Dawson D.V. (2014) Effect of Alveolar Ridge Preservation after Tooth Extraction: A Systematic Review and Meta-analysis. Journal of Dental Research 93: 950-958.

Becker W., Becker B.E. \& Caffesse R. (1994) A comparison of demineralized freeze-dried bone and autologous bone to induce bone formation in human extraction sockets. Journal of Periodontology 65: 1128-1133.

Cardaropoli D., Gaveglio L. \& Cardaropoli G. (2013) Vertical Ridge Augmentation with a Collagen Membrane, Bovine Bone Mineral and Fibrin Sealer: Clinical and Histologic Findings. The International journal of periodontics \& restorative dentistry 33: 583-589.

Cardaropoli G., Araujo M. \& Lindhe J. (2003) Dynamics of bone tissue formation in tooth extraction sites. Journal of clinical periodontology 30: 809-818.

Darby I., Chen S. \& Buser D. (2009) Ridge preservation techniques for implant therapy. The International Journal of Oral \& Maxillofacial Implants 24: 260-271.

Feuille F., Knapp C.I., Brunsvold M.A. \& Mellonig J.T. (2003) Clinical and Histologic Evaluation of Bone-Replacement Grafts in the Treatment of Localized Alveolar Ridge Defects. Part 1: Mineralized Freeze- Dried Bone Allograft. The International journal of periodontics \& restorative dentistry 23: 29-35.

Froum S., Cho S.-C., Rosenberg E., Rohrer M.D. \& Tarnow D.P. (2002) Histological comparison of healing extraction sockets implanted with bioactive glass or demineralized freeze-dried bone allograft: a pilot study. Journal of Periodontology 73 : 94-102.

Hämmerle C.H.F., Araújo M.G., Simion M. \& On Behalf of the Osteology Consensus Group 2011 (2012) Evidence-based knowledge on the biology and treatment of extraction sockets. Clinical Oral Implants Research 23: 80-82.

Johnson K. (1969) A study of the dimensional changes occurring in the maxilla following tooth extraction. Australian Dental Journal 14: 241-244.

Jung R.E., Philipp A., Annen B.M., Signorelli L., Thoma D.S., Hämmerle C.H.F., et al. (2013) Radiographic evaluation of different techniques for ridge preservation after tooth extraction: a randomized controlled clinical trial. Journal of Clinical Periodontology 40: 90-98. 
MacBeth N., Trullenque-Eriksson A., Donos N. \& Mardas N. (2017) Hard and soft tissue changes following alveolar ridge preservation: a systematic review. Clinical Oral Implants Research 28: 982-1004.

Mardas N., Trullenque-Eriksson A., MacBeth N., Petrie A. \& Donos N. (2015) Does ridge preservation following tooth extraction improve implant treatment outcomes: a systematic review: Group 4: Therapeutic concepts \&amp; methods. Clinical Oral Implants Research 26: 180-201.

Meloni S.M., Tallarico M., Lolli F.M., Deledda A., Pisano M. \& Jovanovic S.A. (2015) Postextraction socket preservation using epithelial connective tissue graft vs porcine collagen matrix. 1-year results of a randomised controlled trial. European Journal of Oral Implantology 8: 39-48.

Serino G., Biancu S., lezzi G. \& Piattelli A. (2003) Ridge preservation following tooth extraction using a polylactide and polyglycolide sponge as space filler: a clinical and histological study in humans. Clinical oral implants research 14: 651-658.

Sharan A. \& Madjar D. (2008) Maxillary Sinus Pneumatization Following Extractions: A Radiographic Study. The International Journal of Oral \& Maxillofacial Implants 23: 48-56.

Tan W.L., Wong T.L.T., Wong M.C.M. \& Lang N.P. (2012) A systematic review of post-extractional alveolar hard and soft tissue dimensional changes in humans. Clinical Oral Implants Research 23: 1-21.

Walker C.J., Prihoda T.J., Mealey B.L., Lasho D.J., Noujeim M. \& Huynh-Ba G. (2017) Evaluation of Healing at Molar Extraction Sites With and Without Ridge Preservation: A Randomized Controlled Clinical Trial. Journal of Periodontology 88: 241-249. 


\section{Acknowledgments, source of funding and conflict of interest}

This study was primarily supported by the Clinic of Fixed and Removable Prosthodontics and Dental Material Science, Center of Dental Medicine, University of Zurich, Switzerland and a research grant from Geistlich Pharma AG, Wolhusen, Switzerland. The support of Daniel Wiedemeier, statistician, is highly acknowledged. The authors express their special thanks to Dr. Luca Signorelli (Private practice Zürich, Switzerland) for his support in the assessment of the CBCTs and his constructive contribution to the entire study. The authors report no conflict of interest. 


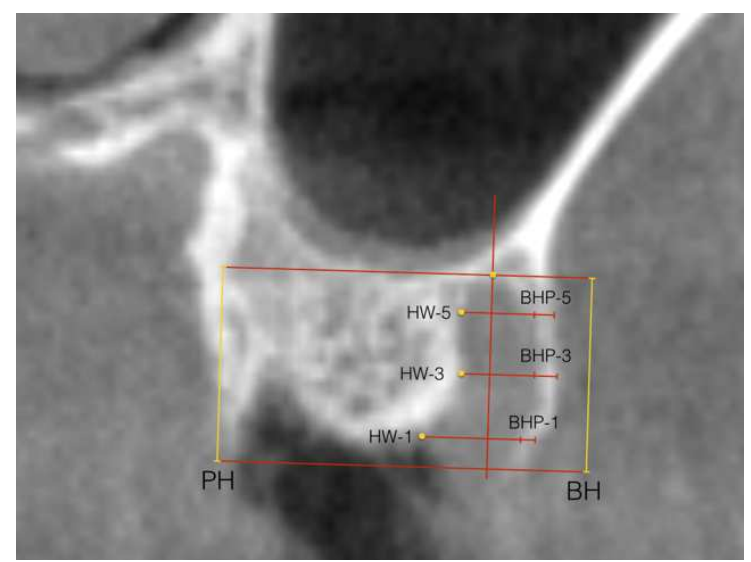

Figure 1. Slice section at baseline. HW-1 (at $1 \mathrm{~mm}$ ), HW-3 (at $3 \mathrm{~mm}$ ) and HW-5 (at $5 \mathrm{~mm}$ ) represent the horizontal width of the extraction socket. $\mathrm{BH}$ and $\mathrm{PH}$ represent the height of the buccal $(\mathrm{BH})$ and palatal $(\mathrm{PH})$ bone plate. BHP-1 (at $1 \mathrm{~mm}), \mathrm{BHP}-3($ at $3 \mathrm{~mm}$ ) and BHP -5 (at $5 \mathrm{~mm}$ ) represent the horizontal width (thickness) of the buccal bone plate.

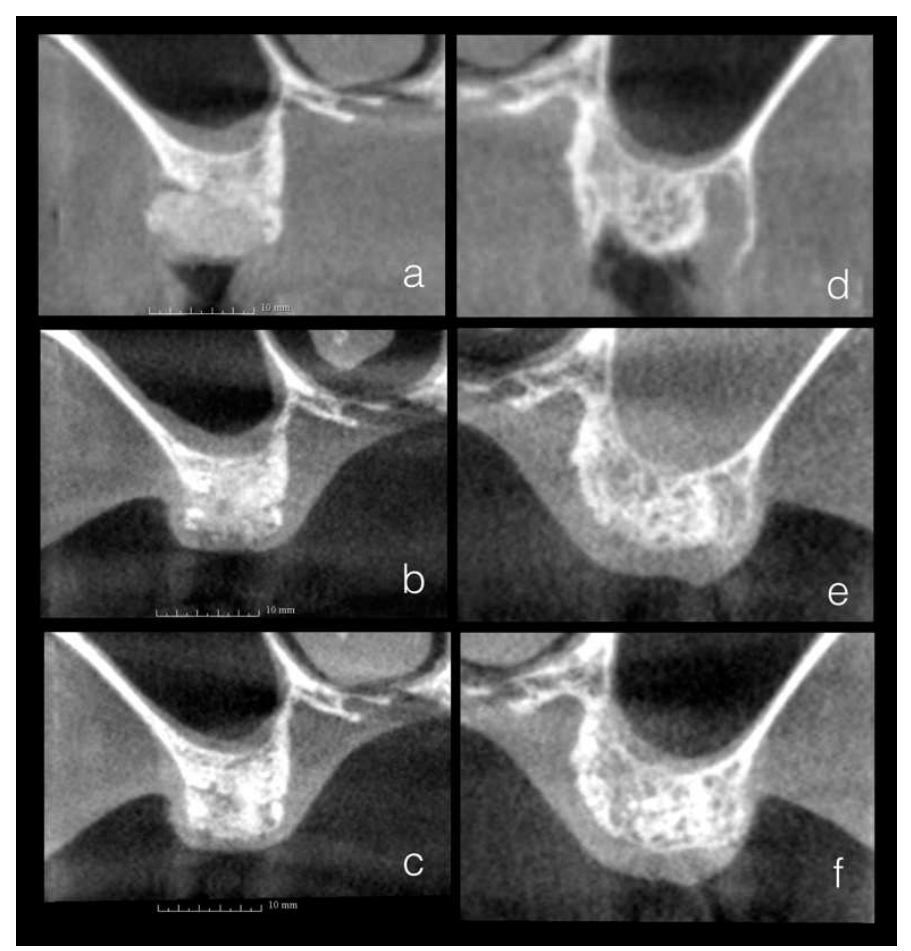

Figure 2. Cone-beam computed tomography (CBCT) of test and control group of different patients at baseline, 3 and 6 months. Test group at baseline (a), 3 months' follow-up (b) and 6 months' follow-up (c). Control group at baseline (d), 3 months' follow-up (e) and 6 months' follow-up (f). 

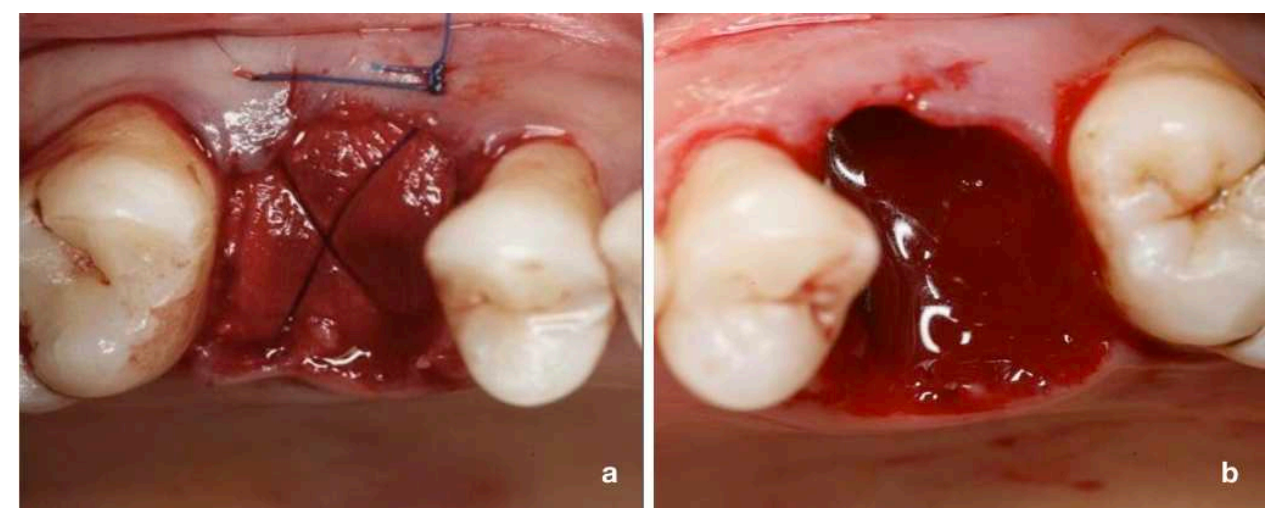

Figure 3. Clinical picture of test group with sockets completely filled with DBBM-C, covered with NBCM and a cross suture keeping the membrane in place (a). Clinical picture of control group without any graft materials and with no sutures (b).
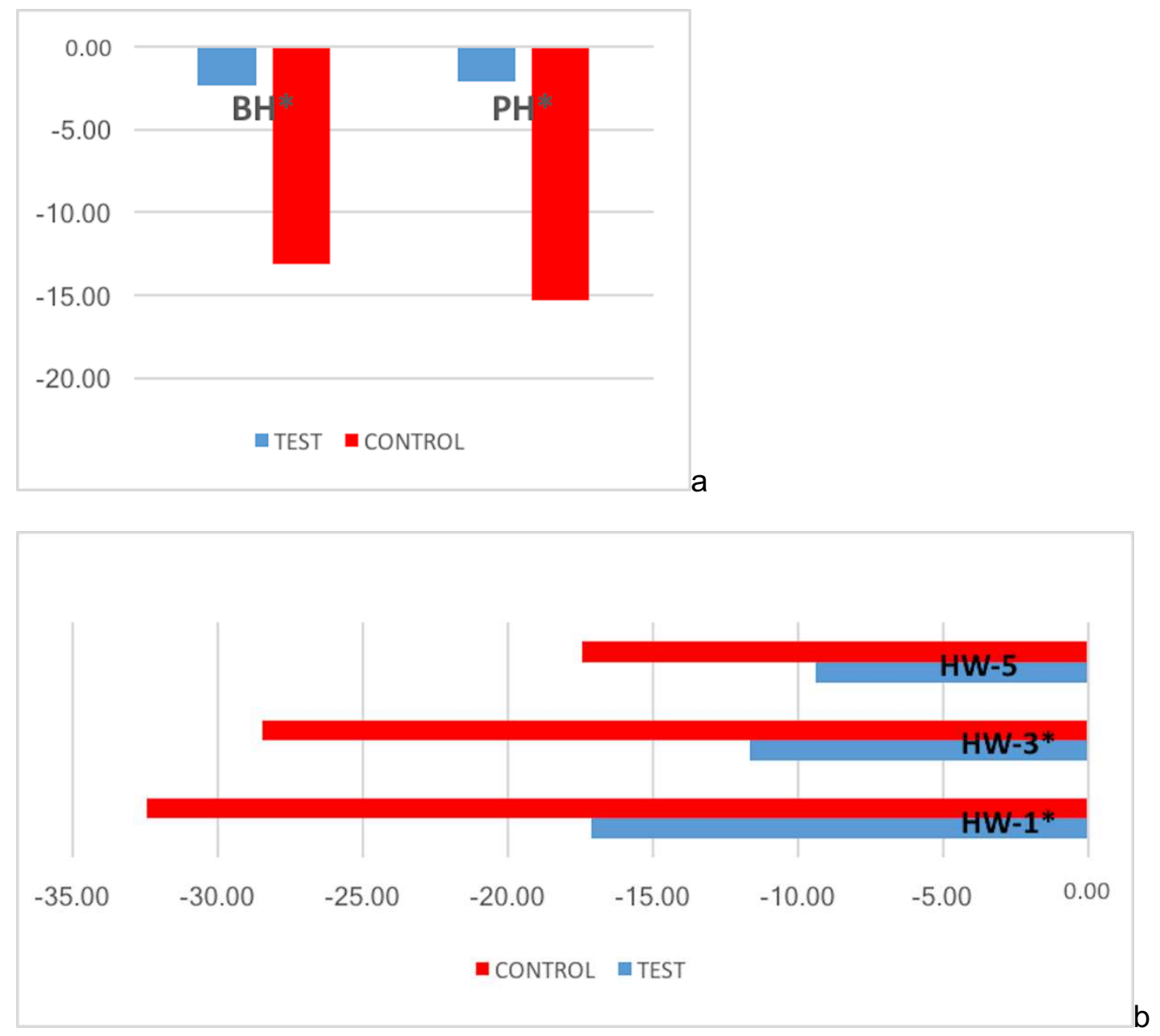
Figure 4. The Relative bone loss in height (\%) over 6 months based on cone-beam computed tomography (CBCT) measurements (a). The Relative bone loss in width (\%) over 6 months based on cone-beam computed tomography (CBCT) measurements (b).

Figure 4b

Relationship between BHP-1 and relative bone loss at:
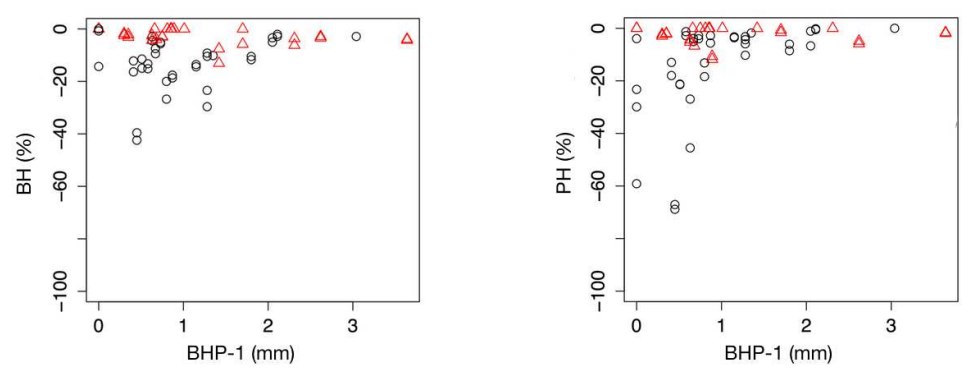

C Control group

$\triangle$ Test group
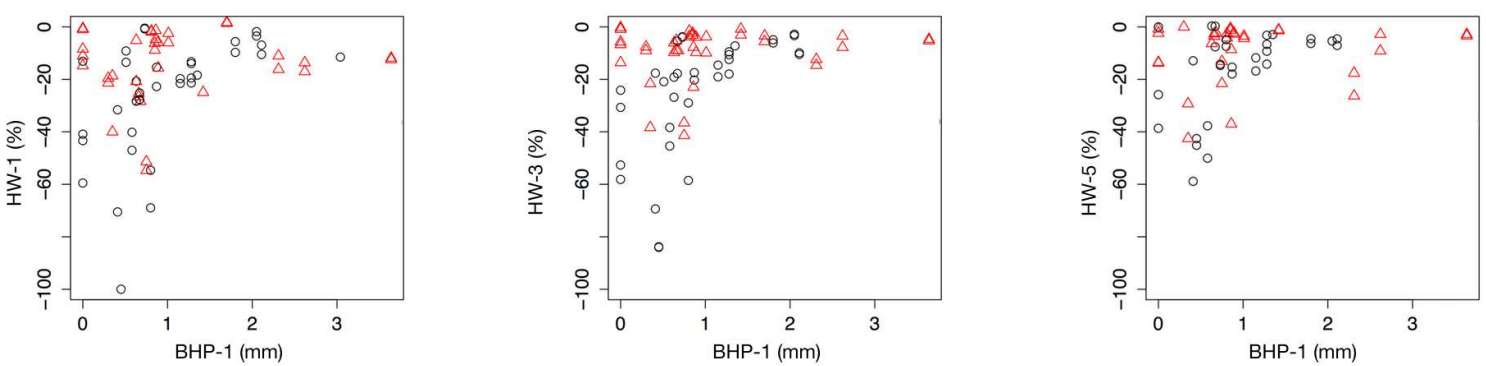

Figure 5. The relationship between BHP-1 and overall bone loss. 